\title{
José MANUEL SÁNChEZ PATRÓN: LOS LíMITES DE LA INVESTIGACIÓN BIOMÉDICA EN EL ORDENAMIENTO JURÍDICO INTERNACIONAL Y EUROPEO ${ }^{1}$
}

\section{LIMITS ON BIOMEDICAL RESEARCH IN INTERNATIONAL AND EUROPEAN LEGAL ORDER}

Resumen. La investigación biomédica ha experimentado un fuerte desarrollo en los últimos años, logrando importantes avances, pero planteado, a su vez, considerables desafíos; en especial, para los derechos humanos. El Derecho internacional y europeo establece los límites necesarios para garantizar los derechos fundamentales del individuo en el desarrollo de la actividad investigadora. El presente trabajo identifica y examina cuáles son esos límites contemplados por los instrumentos internacionales y europeos, al tiempo que los sistematiza y clarifica por medio de una serie de principios que el investigador biomédico deberá observar en el ejercicio de su actividad científica.

\begin{abstract}
Biomedical research has developed strongly in recent years, making significant progress, but raised, in turn, considerable challenges; especially for human rights. The International and European law establishes limits to the research activities in order to guarantee the fundamental rights of the individual. This paper identifies and examines those limits provide by international and european instruments, while systematized and clarified through a set of principles that will have to take into account the biomedical researcher in the exercise of their scientific activity.
\end{abstract}

Palabras clave. Investigación biomédica, Bioderecho, Derecho internacional y Derecho europeo.

Key words. Biomedical research, Biolaw, Internacional law and European law.

\footnotetext{
${ }^{1}$ Profesor Titular de Derecho internacional público y Relaciones internacionales. Departamento de Derecho internacional "Adolfo Miaja de la Muela". Universitat de València. jmsp@uv.es. El presente trabajo tiene su origen en el estudio de nuestra autoría y publicado en su día con el título: Los límites a la investigación genética en el marco jurídico internacional y europeo, en Investigación, Genética y Derecho, (SALCEDO BELTRÁN, C., coord.), Tirant lo Blanch, Valencia, 2008, pp. 239-266.
} 


\section{INTRODUCCIÓN}

Los avances científicos y sus aplicaciones técnicas, experimentados en las últimas décadas, no tienen parangón con los logrados en ningún otro periodo histórico anterior. Este desarrollo ha sido especialmente significativo en el campo de la biomedicina, donde el desciframiento de la secuencia del genoma humano no sólo ha constituido un hito científico, sino un descubrimiento que ha abierto la puerta a nuevas posibilidades. No obstante, todas estas potencialidades conllevan desafíos para el principal beneficiario de estos progresos: el ser humano ${ }^{2}$. La investigación científica es una actividad, en principio, libre ${ }^{3}$. Sin embargo, sus resultados y, sobre todo, su puesta en práctica pueden conllevar abusos que desemboquen en violaciones de los derechos fundamentales. Ante esta eventualidad, el Derecho se erige en una de las principales herramientas que pueden ser empleadas para evitar esos excesos y corregirlos en caso de que se produzcan, garantizando con ello la dignidad humana ${ }^{4}$.

Aparte de las soluciones previstas en los respectivos ordenamientos jurídicos internos, el Derecho internacional también contribuye al cumplimiento de la función de limitar la actividad investigadora con el fin de salvaguardar los derechos inalienables del ser humano. Sin embargo, la mayoría de los instrumentos internacionales existentes al respecto no son normas jurídicas en sentido estricto, sino textos de carácter no vinculante con los que se quiere llamar la atención de los Estados para que adopten las medidas internas pertinentes ${ }^{5}$. Esta circunstancia explica que los instrumentos

\footnotetext{
${ }^{2}$ FOSTER, CH., Human dignity in bioethics and law, Hart, Oxford..., 2011.

${ }^{3}$ El artículo 15.3 del Pacto Internacional de Derechos Económicos, Sociales y Culturales, de 16 de diciembre de 1966, establece que: "Los Estados Partes en el presente Pacto se comprometen a respetar la indispensable libertad para la investigación científica y para la actividad creadora". También, el artículo 15 del Convenio para la protección de los derechos humanos y de la dignidad del ser humano respecto a las aplicaciones de la biología y de la medicina, de 4 de abril de 1997, dispone que: "La investigación científica en el ámbito de la biología y la medicina se efectuará libremente (...)". Por su parte, en el ámbito de la Unión Europea, la Carta de Derechos Fundamentales de la Unión Europea, de 12 de diciembre de 2007, afirma en su artículo 13 que: "Las artes y la investigación científica son libres".

${ }^{4}$ WYNDHAM, J.M., WEIGERS VITULLO, M., The right to science - whose right? To what? European Journal of Human Rights, vol. 4, 2015, pp. 431-461, y BESSON, S., Science without borders and the boundaries of human rights: who owes the human right to science?, Ibid., pp. 462-485.

${ }^{5}$ ADORNO, R., Principles of international biolaw. Seeking common ground at the intersection of bioethics and human rights, Bruylant, Bruxelles, 2013, pp. 37 y ss.
} 
IUS ET SCIENTIA, (ISSN: 2444-8478) 2016, Vol.2, nº 1, pp.35-58

JOSÉ MANUEL SÁNCHEZ PATRÓN: LOS LÍMITES DE LA INVESTIGACIÓN BIOMÉDICA EN EL ORDENAMIENTO JURÍDICO INTERNACIONAL Y EUROPEO

FECHA DE ENVÍO DE ORIGINAL: 1.02.2016 FECHA DE ACEPTACIÓN: 1.03.2016

DOI: $\underline{\text { http://dx.doi.org/10.12795/IETSCIENTIA.2016.i01.03 }}$

internacionales consultados están formulados de forma genérica al tiempo que su eficacia no se encuentra asegurada. Los Estados son los que pueden hacer realidad en su ámbito interno los deseos expresados en el internacional.

Antes de examinar los instrumentos internacionales aplicables a la investigación científica en el ámbito biomédico e identificar cuáles son los límites dispuestos para el desarrollo adecuado de la misma, resulta necesario delimitar que debe entenderse por investigación científica en este contexto. La lectura de dichos textos internacionales nos permite deducir que la investigación científica es aquella actividad que, mediante el cumplimiento de ciertas condiciones y con independencia de sus modalidades, tiene por objetivo adquirir conocimientos a fin de mejorar la salud y el bienestar de los seres humanos. Esta actividad puede consistir en una intervención sobre el "material humano" del individuo ${ }^{6}$, así como en una interacción con la "información identificable" que se obtenga del mismo ${ }^{7}$. La actividad científica no sólo está conformada únicamente por la extracción de muestras biológicas del sujeto intervenido sino también por las informaciones personales que se obtengan de dicho individuo ${ }^{8}$. Sin embargo, lo que convierte esta actividad en científica es su adecuación a una metodología determinada y la consecución de una finalidad específica con arreglo a las cuales se lleva a efecto. Concretamente, esta finalidad específica no es otra que la de avanzar en la comprensión de la etiología/patogenia de las enfermedades y en el desarrollo de los procedimientos preventivos, diagnósticos o terapéuticos - para tratarlas, con el objetivo final de mejorar la calidad/esperanza de vida de la humanidad ${ }^{9}$. Esto quiere decir que cualquier actividad, aunque pueda aparentemente revestir el carácter de científica, no debe considerarse como tal si no satisface la finalidad que acaba de apuntarse. La falta de

\footnotetext{
${ }^{6}$ VAN ASSCHE, K., STERCKZ, S., The protection of human dignity in research involving human body material, en "Humanity across international law and biolaw" (VAN BEERS, B., CORRIAS, L., WERNER, W., ed.), Cambridge University Press, Cambridge, 2014, pp. 265-287 y EBOLI, V., The new ethical and legal frontiers in the use and storage of human tissues for reserch, Aracne, Roma, 2013.

${ }^{7}$ LENK, CH., Human tissue research: an european perspective on the ethical and legal challenges (LENK, CH., y otros, ed.), Oxford University Press, New York..., 2011.

${ }^{8}$ Declaración de Helsinki de la Asociación Médica Mundial de junio de 1964 (A.1).

${ }^{9}$ Declaración de Helsinki de la Asociación Medica Mundial de junio de 1964 (A.6). También, Declaración Universal sobre el Genoma Humano y los Derechos Humanos de 11 de noviembre de 1997 (Art. 12b).Esta última Declaración internacional ha sido hecha suya por la Asamblea General de la ONU. Ver: A/RES/53/152 de 10 de marzo de 1999.
} 
IUS ET SCIENTIA, (ISSN: 2444-8478) 2016, Vol.2, $\mathrm{n}^{\circ} 1$ 1, pp.35-58

JOSÉ MANUEL SÁNCHEZ PATRÓN: LOS LÍMITES DE LA INVESTIGACIÓN BIOMÉDICA EN EL ORDENAMIENTO JURÍDICO INTERNACIONAL Y EUROPEO

FECHA DE ENVÍO DE ORIGINAL: 1.02.2016 FECHA DE ACEPTACIÓN: 1.03 .2016

DOI: $\underline{\text { http://dx.doi.org/10.12795/IETSCIENTIA.2016.i01.03 }}$

cumplimiento de este objetivo último hace que ciertas actividades no puedan considerarse como científicas strictu sensu ${ }^{10}$.

A la vista de lo anterior, y una vez delimitada la noción de investigación científica en el ámbito biomédico, podemos centrarnos en el examen de los límites que deben ser tenidos en cuenta en el momento de llevarla a cabo, así como los que deben observarse con respecto a quienes participen en la misma. A estos efectos, nos referiremos, en primer lugar, a los límites relativos a la conducción de la investigación biomédica, para, en segundo lugar, centrarnos en los límites concernientes a la participación de los sujetos intervinientes en dicha actividad investigadora. Ambos serán deducidos del análisis de los instrumentos internacionales existentes y se expondrán en forma de principios con el fin de sintetizar y clarificar el contenido de cada uno de ellos.

\section{LOS LÍMITES MATERIALES: LOS PRINCIPIOS APLICABLES A LA CONDUCCIÓN DE LA INVESTIGACIÓN BIOMÉDICA}

El principio de oportunidad. La Recomendación (1990)3 del Comité de Ministros del Consejo de Europa acerca de la investigación médica sobre el ser humano, de 6 de febrero de 1990, señala que: “(t)oda investigación médica proyectada que no responda a los criterios científicos en su concepción y que no responda a las cuestiones planteadas es inaceptable, incluso si la manera en la que debe ser efectuada no presenta ningún riesgo para la persona que se presta a la investigación" ${ }^{11}$. Esto significa que la investigación proyectada deberá ser pertinente desde el punto de vista científico y encontrarse convenientemente justificada teniendo en cuenta el objetivo que se haya

\footnotetext{
${ }^{10}$ Así, por ejemplo, no cabe considerar como investigación científica las prácticas médicas que ya se encuentren aceptadas, aunque presenten un carácter innovador. Tampoco aquellas que, pese a poseer esta última característica, no supongan realmente un progreso en los términos en los que lo hemos concebido con anterioridad. Por supuesto que no podrá incluirse dentro de este tipo de actividad aquella que esté destinada a mejorar la salud o el bienestar de un paciente en particular y no de los individuos en general. Sólo revestirá el carácter de investigación científica la realizada con el ánimo de alcanzar un beneficio para el conjunto de la humanidad, aunque luego resulte aplicable únicamente a individuos concretos. Y por último, la recopilación/tratamiento de datos con fines exclusivamente estadísticos tampoco puede ser equiparada a la actividad realizada con una finalidad investigadora. Al respecto, ver: Informe explicativo al Protocolo Adicional al Convenio sobre los Derechos Humanos y la Biomedicina relativo a la investigación biomédica de 25 de enero de 2005 .

${ }^{11}$ Principio 11.
} 
IUS ET SCIENTIA, (ISSN: 2444-8478) 2016, Vol.2, nº 1, pp.35-58

JOSÉ MANUEL SÁNCHEZ PATRÓN: LOS LÍMITES DE LA INVESTIGACIÓN BIOMÉDICA EN EL ORDENAMIENTO JURÍDICO INTERNACIONAL Y EUROPEO

FECHA DE ENVÍO DE ORIGINAL: 1.02.2016 FECHA DE ACEPTACIÓN: 1.03 .2016

DOI: $\underline{\text { http://dx.doi.org/10.12795/IETSCIENTIA.2016.i01.03 }}$

propuesto alcanzar con su ejecución ${ }^{12}$. Además, dicha investigación deberá contemplar las reglas profesionales aplicables al tipo de investigación desarrollada así como las directrices oportunas para que puedan ser éticamente aceptables ${ }^{13}$.

El cumplimiento de estas exigencias generales será supervisado por los llamados comités de ética a los que también se refieren de forma expresa algunos textos internacionales ${ }^{14}$. Estos mismos instrumentos mencionan cuáles son los requisitos mínimos que deben reunir estos órganos de supervisión para realizar su labor sobre las propuestas que se les someta a su consideración. Por regla general, estas instituciones deben presentar una composición independiente, multidisciplinaria, pluralista y capacitada para valorar, en particular, los aspectos éticos y jurídicos que se deriven de cada uno de los proyectos de investigación que vaya a supervisar ${ }^{15}$.

El principio de preeminencia. Desde la Declaración de Helsinki de la Asociación Médica Mundial de junio de 1964 hasta la Declaración Universal sobre Bioética y Derechos Humanos de 19 de octubre de 2005, se subraya la necesidad de que el interés del individuo prime sobre el de la ciencia y la sociedad ${ }^{16}$. Concretamente, el Convenio relativo a los Derechos Humanos y la Biomedicina de 4 de abril de 1997 establece como disposición general que: “(e)l interés y el bienestar del ser humano deberán prevalecer

\footnotetext{
${ }^{12}$ DE LEUCONA, I., Régimen jurídico de la investigación biomédica en terapia celular y medicina regenerativa desde una perspectiva bioética, en "Marco jurídico europeo relativo a la investigación biomédica en transferencia nuclear y reprogramación celular" (GARCÍA SAN JOSÉ, D.I., coord.), Aranzadi, Cizur Menor (Navarra), 2012, pp. 67-92.

${ }^{13}$ Ver, por ejemplo, El Convenio sobre los Derechos Humanos y la Biomedicina de 4 de abril de 1997 (art.15iii) y su Protocolo Adicional de 25 de enero de 2005 (arts. 7 y 8). El primero de los instrumentos internacional fue firmado por España el 4 de abril de 1997 y ratificado el 1 de septiembre de 1999. Entró en vigor para nuestro país el 1 de enero de 2000. B.O.E. n ${ }^{\circ} 251$ de 20 de octubre de 1999. Sin embargo, el Protocolo adicional de 25 de enero de 2005 sobre la investigación biomédica no ha sido firmado y ratificado por España por lo que, consiguientemente, no se encuentra en vigor para nuestro país.

${ }^{14}$ El Grupo de Especialistas en Investigación Biomédica (CDBI-CO-GT2) dependiente del Comité Director de la Bioética del Consejo de Europa ha elaborado una Guía para los Miembros de los Comités de Ética de Investigación. Este documento ha sido diseñado para "ayudar a los Comités de Ética de Investigación en la realización de su importante papel como evaluadores de las propuestas de investigación que involucran a seres humanos". Guía para los Miembros de los Comités de Ética de Investigación, Comité Director de la Bioética, Consejo de Europa, Estrasburgo, 2012, p. 7. Su borrador fue sometido a consulta y se revisó a la luz de los comentarios recibidos. El texto definitivo fue aprobado por el Comité Director de Bioética el 3 de diciembre de 2010. El texto del documento se encuentra disponible en diferentes idiomas - entre ellos, el castellano - en la siguiente dirección web (consultada en febrero de 2016):http://www.coe.int/en/web/bioethics/guide-for-research-ethics-committees-members

${ }^{15}$ Ver, por ejemplo, El Protocolo Adicional al Convenio sobre los Derechos Humanos y la Biomedicina de 25 de enero de 2005 (arts. 7 y 8). Este mismo instrumento internacional añade en su anexo una propuesta de la información que debe quedar incorporada en los proyectos de investigación con vistas a su consideración por parte de los respectivos comités éticos de los Estados Partes.

${ }^{16}$ A.5 y art. 3.2, respectivamente.
} 
IUS ET SCIENTIA, (ISSN: 2444-8478) 2016, Vol.2, nº 1, pp.35-58

JOSÉ MANUEL SÁNCHEZ PATRÓN: LOS LÍMITES DE LA INVESTIGACIÓN BIOMÉDICA EN EL ORDENAMIENTO JURÍDICO INTERNACIONAL Y EUROPEO

FECHA DE ENVÍO DE ORIGINAL: 1.02.2016 FECHA DE ACEPTACIÓN: 1.03 .2016

DOI: $\underline{\text { http://dx.doi.org/10.12795/IETSCIENTIA.2016.i01.03 }}$

sobre el interés exclusivo de la sociedad o de la ciencia" ${ }^{" 17}$. Esta prevalencia del interés de la persona sobre el de la ciencia y la sociedad explica que cualquier investigación que pretenda llevarse a cabo deberá estar supeditada al respeto del ser humano que participe en la misma ${ }^{18}$. Y ello pese a la eventual trascendencia científica y social que puedan tener sus resultados. Aunque la investigación proyectada sea, con carácter general, beneficiosa para el progreso de la ciencia así como para el bienestar de la sociedad, ello no constituye una justificación suficiente para poner en riesgo a los participantes ${ }^{19}$. En última instancia, caso de que los resultados de la investigación proyectada sólo redunden en beneficio de la ciencia o la sociedad, pero no directamente de los participantes, éstos últimos no serán sometidos a un riesgo que vaya más allá de lo que puede ser considerado como asumible ${ }^{20}$.

Este principio, como el resto de los principios enunciados en el presente trabajo, encuentra su anclaje último en el respeto de la dignidad humana al que debe acomodarse la investigación científica. Cualquier investigación proyectada, como punto de partida, debe ser respetuosa con los derechos fundamentales de la persona. Al respecto, el Convenio relativo a los Derechos Humanos y la Biomedicina, de 4 de abril de 1997, nos recuerda que "la investigación científica en el ámbito de la biología y de la medicina se efectuará (...) a reserva de lo dispuesto en el presente Convenio y en otras disposiciones jurídicas que garanticen la protección del ser humano" ${ }^{\text {21 }}$. Esta limitación de alcance general está presente, de un modo u otro, en todos los instrumentos internacionales relativos a la investigación biomédica ${ }^{22}$.

\footnotetext{
${ }^{17}$ Art.2.

${ }^{18}$ Guía para los Miembros de los Comités de Ética de Investigación, op.cit., p., 10.

${ }^{19}$ DONDERS, Y., Balancing interests: limitations to the right to enjoy the benefits of scientific progress and its applications, en European Journal of Human Rights, vol. 4, 2015, pp. 486-503.

${ }^{20}$ Guía para los Miembros de los Comités de Ética de Investigación, op.cit., p. 35.

${ }^{21}$ Art. 15.

${ }^{22}$ En particular, y en relación con el genoma humano, el artículo 10 de la Declaración Universal sobre el Genoma Humano y los Derechos Humanos, de 11 de noviembre de 1997, señala que: "(n)inguna investigación relativa al genoma humano ni ninguna de sus aplicaciones, en particular en las esferas de la biología, la genética y la medicina, podrá prevalecer sobre el respeto de los derechos humanos, de las libertades fundamentales y de la dignidad humana de los individuos o, si procede, de grupos de individuos". Sobre esta base, el Convenio relativo a los Derechos Humanos y la Biomedicina, de 4 de abril de 1997, prohíbe la introducción de modificaciones en el genoma de la descendencia en su artículo 13, mientras que el artículo 11 de la misma Declaración Universal sobre el Genoma Humano y los Derechos Humanos, de 11 de noviembre de 1997, prohíbe la clonación de seres humanos con fines reproductivos. Ésta última prohibición ha sido objeto de una Declaración específica en el marco universal, adoptada por la Asamblea General de la ONU el 8 de marzo de 2005. A/RES/59/280 de 23 de marzo de 2005 y Anexo (España votó en contra). En el ámbito regional, existe una referencia expresa al
} 
IUS ET SCIENTIA, (ISSN: 2444-8478) 2016, Vol.2, nº 1, pp.35-58

JOSÉ MANUEL SÁNCHEZ PATRÓN: LOS LÍMITES DE LA INVESTIGACIÓN BIOMÉDICA EN EL ORDENAMIENTO JURÍDICO INTERNACIONAL Y EUROPEO

FECHA DE ENVÍO DE ORIGINAL: 1.02.2016 FECHA DE ACEPTACIÓN: 1.03 .2016

DOI: $\underline{\text { http://dx.doi.org/10.12795/IETSCIENTIA.2016.i01.03 }}$

El principio de proporcionalidad. Corolario del anterior es el principio de seguridad.

La Declaración de Helsinki de la Asociación Médica Mundial de junio de 1964 dispone que los "beneficios previsibles" de la investigación proyectada deben ser mayores que los "riesgos calculados". Por "riesgo calculado" se entiende aquel que se deriva de la investigación en sí, unido al coste que puede acarrear para los participantes en la misma, máxime - el texto declarativo así lo establece - si tales participantes son individuos $\operatorname{sanos}^{23}$. Este instrumento internacional se conforma con exigir que el beneficio esperado sea, simplemente, "mayor" que el riesgo asumido. Sin embargo, los textos internacionales posteriores - especialmente el Convenio relativo a los Derechos Humanos y la Biomedicina de 4 de abril de 1997 y su Protocolo Adicional relativo a la investigación biomédica de 25 de enero de $2005^{24}$ - son más laxos con respecto al nivel de exigencia y admiten que el participante sea sometido a un mayor riesgo siempre y cuando esta mayor exposición lleve aparejada un previsible beneficio directo para él ${ }^{25}$.

Esto quiere decir que la necesidad de que el beneficio obtenido sea superior al riesgo soportado es sustituido por un criterio menos exigente: La investigación proyectada podrá realizarse si los resultados esperados no son "desproporcionados" en relación con el riesgo asumido por quienes participan en la misma ${ }^{26}$. En estas circunstancias, la ejecución del proyecto de investigación sólo deberá llevarse a cabo "si los riesgos previsibles no son desproporcionados frente a los beneficios potenciales" por lo que todos ellos deberían "someterse a una cuidadosa ponderación del balance riesgo /

respecto en el artículo 3 d) de la Carta de Derechos Fundamentales de la Unión Europea, de 7 de diciembre de 2000, y un tratado internacional exclusivo: el Protocolo Adicional al Convenio relativo a los Derechos Humanos y la Biomedicina, relativo a la Prohibición de la Clonación de Seres Humanos de fecha 12 de enero de 1998. España lo firmó el 12 de enero de 1998 y lo ratificó el 24 de enero de 2000. Entró en vigor para nuestro país el 1 de marzo de 2001. B.O.E. n ${ }^{\circ} 52$ de 1 de marzo de 2001, pp. 7671 y ss.

23 El art. 6.2 del Protocolo Adicional al Convenio sobre los Derechos Humanos y la Biomedicina, de 25 de enero de 2005, dispone que el individuo "sano" no debe ser sometido a riesgo ni a ningún tipo de coacción inaceptable.

${ }^{24}$ Arts. 16 ii) y 6.1 , respectivamente.

${ }^{25}$ Guía para los Miembros de los Comités de Ética de Investigación, op.cit., p. 35.

${ }^{26}$ No obstante, los dos tratados internacionales, a diferencia de lo previsto por la Declaración de Helsinki, establecen que el proyecto investigador no podrá llevarse a la práctica a no ser que: “(...) no exista un método alternativo al experimento con seres humanos de eficacia comparable". Arts. 16i) y 5, respectivamente. Al respecto, también ver: Guía para los Miembros de los Comités de Ética de Investigación, op.cit., p. 11. 
IUS ET SCIENTIA, (ISSN: 2444-8478) 2016, Vol.2, nº 1, pp.35-58

JOSÉ MANUEL SÁNCHEZ PATRÓN: LOS LÍMITES DE LA INVESTIGACIÓN BIOMÉDICA EN EL ORDENAMIENTO JURÍDICO INTERNACIONAL Y EUROPEO

FECHA DE ENVÍO DE ORIGINAL: 1.02.2016 FECHA DE ACEPTACIÓN: 1.03 .2016

DOI: $\underline{\text { http://dx.doi.org/10.12795/IETSCIENTIA.2016.i01.03 }}$

beneficio" ${ }^{27}$. Los riesgos no sólo incluyen los de naturaleza física o psicológica de los participantes sino también los "sociales y económicos"28.

En esa misma línea, la Recomendación (2006)4 del Comité de Ministros del Consejo de Europa sobre la investigación utilizando material biológico de origen humano de 15 de marzo de 2006, afirma que: “(1)os riesgos para las personas concernidas y en su caso, para su familia, vinculados a las actividades de investigación, en particular para la vida privada, deberían reducirse al mínimo en relación con las características de la actividad investigadora. Además estos riesgos no deberían ser desproporcionados en relación con el beneficio potencial de las actividades de investigación. Los riesgos eventuales para los individuos que forman parte del mismo grupo que la persona afectada, deberían ser igualmente tomados en consideración en este contexto"29.

El principio de accesibilidad. Los resultados de investigación llevada a efecto deben ser accesibles, en primer lugar, a los propios participantes en la misma y, en segundo lugar, a la sociedad en general ${ }^{30}$. Por lo que respecta al primer aspecto de este principio, el Protocolo Adicional al Convenio sobre los Derechos Humanos y la Biomedicina de 25 de enero de 2005, señala que: “(1)as personas participantes en una investigación tienen el derecho de conocer cualquier información recogida sobre su salud"31 al tiempo que, en aplicación del Convenio relativo a los Derechos Humanos y la Biomedicina de 4 de abril de 1997, también tienen derecho a no recibir dicha información ${ }^{32}$. Además, “(s)i lo solicitan, los participantes deben tener acceso a las conclusiones de la investigación en un plazo razonable" ${ }^{33}$, y en una forma comprensible ${ }^{34}$. Con respecto a esta última cuestión, la información que vaya a facilitarse a los participantes a resultas de la investigación realizada deberá "hacerse dentro de un entorno de asistencia o asesoramiento médico de modo que profesionales de la salud puedan explicar la

\footnotetext{
${ }^{27}$ Guía para los Miembros de los Comités de Ética de Investigación, op.cit., p. 12.

${ }^{28}$ Ibid.

${ }^{29}$ Art. 5.

${ }^{30}$ SHAVER, L., The right to science: ensuring that everyone benefits from scientific and technological progress, en European Journal of Human Rights, vol. 4, 2015, pp. 411-430.

${ }^{31}$ Art. 26.1.

${ }^{32}$ Art. 10. Al respecto, ver también: TORRES CAZORLA, M.I., Legislación interna española y biomedicina: una aproximación a los recientes avances experimentados respecto al derecho a ser/no ser informado, en Bioderecho internacional y europeo: desafíos actuales (SANCHEZ PATRON, J.M., coord.), Tirant lo Blanch, Valencia, 2014, pp. 41-66.

${ }^{33}$ Art. 28.2.

${ }^{34}$ Guía para los Miembros de los Comités de Ética de Investigación, op.cit., p. 42.
} 
IUS ET SCIENTIA, (ISSN: 2444-8478) 2016, Vol.2, nº 1, pp.35-58

JOSÉ MANUEL SÁNCHEZ PATRÓN: LOS LÍMITES DE LA INVESTIGACIÓN BIOMÉDICA EN EL ORDENAMIENTO JURÍDICO INTERNACIONAL Y EUROPEO

FECHA DE ENVÍO DE ORIGINAL: 1.02.2016 FECHA DE ACEPTACIÓN: 1.03 .2016

DOI: http://dx.doi.org/10.12795/IETSCIENTIA.2016.i01.03

naturaleza y el alcance de los resultados de un modo que sea fácilmente comprensible para los participantes, así como discutir las opciones disponibles para la prevención, tratamiento u otras actuaciones posibles" 35 . Esta información se facilitará al participante de forma confidencial ${ }^{36}$.

Ahora bien, este derecho a conocer la información existente en relación con la salud del participante, tiene un alcance diferente si la investigación proyectada se realiza con “información identificable" en vez de "material humano". En el primer supuesto, la Declaración Internacional sobre los Datos Genéticos de 19 de septiembre de 2003 reconoce el derecho a acceder a los resultados de la investigación en el caso de que la persona sea identificable a partir de la información genética suministrada para la ejecución de la investigación. Este derecho - siempre según la Declaración Internacional comentada - se extendería a los "familiares" del participante en la investigación proyectada, dado que éstos, como es sabido, podrían verse afectados por la aportación de información genética que se hubiera realizado. Ahora bien, fuera de este caso en el que la información delata a quien participa en la investigación - y por extensión a sus familiares -, no parece que el derecho a ser informado de los resultados de la investigación deba también serle reconocido a los individuos que no se exponen a ser identificados con la información genética proporcionada en dicha investigación ${ }^{37}$. Esta falta de reconocimiento obedece realmente a una imposibilidad de carácter material: la anonimización de la información empleada en este tipo de investigación proyectada hará "imposible informar de cualquier resultado relacionado con la (misma) que pudiera afectar a la salud del" participante "anónimo"38.

En relación con el acceso de la sociedad a los resultados de la investigación, la Declaración Universal sobre el Genoma Humano y los Derechos Humanos de 11 de noviembre de 1997, recoge como disposición general que "toda persona" debe tener acceso a los progresos realizados en el ámbito de genética entre otros ${ }^{39}$, lo que conlleva

\footnotetext{
${ }^{35}$ Guía para los Miembros de los Comités de Ética de Investigación, op.cit., p. 41.

36 Ibid.

${ }^{37}$ Art. 10.

${ }^{38}$ Guía para los Miembros de los Comités de Ética de Investigación, op.cit., p. 40.

${ }^{39}$ Art. 12a).
} 
IUS ET SCIENTIA, (ISSN: 2444-8478) 2016, Vol.2, nº 1, pp.35-58

JOSÉ MANUEL SÁNCHEZ PATRÓN: LOS LÍMITES DE LA INVESTIGACIÓN BIOMÉDICA EN EL ORDENAMIENTO JURÍDICO INTERNACIONAL Y EUROPEO

FECHA DE ENVÍO DE ORIGINAL: 1.02.2016 FECHA DE ACEPTACIÓN: 1.03 .2016

DOI: $\underline{\text { http://dx.doi.org/10.12795/IETSCIENTIA.2016.i01.03 }}$

la difusión pública de los resultados obtenidos a través de los medios pertinentes ${ }^{40}$; bien entendido que esta obligación de publicar los resultados de la investigación obtenidos, incluye tanto los favorables como los "desfavorables"41. Esta misma disposición se encuentra recogida en la Declaración Universal sobre Bioética y Derechos Humanos de

19 de octubre de $2005^{42}$; si bien, este último instrumento internacional incorpora entre sus cláusulas generales, una que desarrolla la máxima anterior. La disposición en cuestión, enunciada como "aprovechamiento compartido de los beneficios", afirma lo siguiente: "(1)os beneficios resultantes de toda investigación científica y sus aplicaciones deberían compartirse con la sociedad en su conjunto y en el seno de la comunidad internacional, en particular con los países en vías en desarrollo. Los beneficios que se deriven de la aplicación de este principio podrán revestir las siguientes formas: e) acceso a los conocimientos científicos y tecnológicos" ${ }^{\prime 4}$.

El principio de resarcibilidad. El Protocolo Adicional al Convenio sobre los Derechos Humanos y la Biomedicina de 25 de enero de 2005 es un tratado internacional - se trata, por tanto, de un instrumento vinculante - que se encuentra especializado en la regulación de la investigación científica. Sin embargo, ninguna disposición de su contenido prevé la obligación de indemnizar a los participantes en una investigación por los daños o perjuicios que les puede ocasionar la misma ${ }^{44}$. Sólo el Convenio sobre los Derechos Humanos y la Biomedicina de 4 de abril de 1997, del que éste Protocolo Adicional trae causa, establece, con carácter general que: “(1)a persona que haya sufrido

\footnotetext{
${ }^{40}$ Guía para los Miembros de los Comités de Ética de Investigación, op.cit., p. 41.

${ }^{41}$ Los equipos de investigación pueden verse conminados a no publicar resultados desfavorables o bien a publicar una información sesgada de los mismos. Esta circunstancia podría impedir el general conocimiento de investigaciones ya efectuadas, o lo que es, aún peor, la no difusión de todos los resultados obtenidos en la misma lo que puede perturbar sus aplicaciones prácticas. Para evitar estos efectos, la Guía para los Miembros de los Comités de Ética de Investigación propone la creación de un registro de proyectos de investigación biomédica y la obligación de inscribir en el mismo cualquier investigación proyectada permitiendo el acceso público al contenido del mismo. Op.cit., p. 42.

${ }^{42}$ Art. 2f).

${ }^{43}$ Art. 15.1e). También ver: Grupo Europeo de Ética de las Ciencias y de Nuevas Tecnologías de la Comisión Europea. Opinión $\mathrm{n}^{\circ} 17$ sobre los aspectos éticos de la investigación clínica en los Países en desarrollo de 4 de febrero de 2003.

${ }^{44}$ Aunque los accidentes producidos como consecuencia de la participación de individuos (sanos) son excepcionales, las hemerotecas ofrecen noticias de este tipo de accidentes. Al respecto, tuvo bastante eco en la prensa internacional el accidente producido en el Reino Unido cuando seis jóvenes y sanos que probaron con un anticuerpo para el tratamiento de la leucemia presentaron fallos multi-orgánico. The Guardian de 17 de marzo de 2006. Recientemente, también, seis personas sanas, sometidas a un ensayo de un fármaco anti-ansiedad en Francia, resultaron afectados con trastornos neurológicos graves (uno de ellos con muerte cerebral). Ver: Diario La Vanguardia de 16 de enero de 2016, p. 35.
} 
IUS ET SCIENTIA, (ISSN: 2444-8478) 2016, Vol.2, nº 1, pp.35-58

JOSÉ MANUEL SÁNCHEZ PATRÓN: LOS LÍMITES DE LA INVESTIGACIÓN BIOMÉDICA EN EL ORDENAMIENTO JURÍDICO INTERNACIONAL Y EUROPEO

FECHA DE ENVÍO DE ORIGINAL: 1.02.2016 FECHA DE ACEPTACIÓN: 1.03.2016

DOI: http://dx.doi.org/10.12795/IETSCIENTIA.2016.i01.03

un daño justificado como resultado de una intervención tendrá derecho a una reparación equitativa en las condiciones y modalidades previstas en la ley"(la cursiva es nuestra) ${ }^{45}$. Aunque el texto de la disposición normativa reproducida no se refiere a la investigación científica en particular, creemos que el término "intervención” permitiría incluir este tipo de actividades en su ámbito de aplicación ${ }^{46}$.

No obstante, varias declaraciones y resoluciones de órganos internacionales y europeos sí recogen este principio de resarcibilidad en sus actos institucionales. Sin ir más lejos, la Declaración Universal sobre el Genoma Humano y los Derechos Humanos de 11 de noviembre de 1997 dispone que: “(t)oda persona tendrá derecho, de conformidad con el derecho internacional y el derecho nacional, a una reparación equitativa de un daño del que pueda haber sido víctima, cuya causa directa y determinante pueda haber sido una intervención en su genoma"47. En este mismo sentido, aunque referido a la investigación científica en general y circunscrita al ámbito territorial específicamente europeo, la Recomendación (1990)3 del Comité de Ministros del Consejo de Europa sobre la investigación médica sobre el ser humano de 6 de febrero de 1990, establece, en su principio 14, lo siguiente: “(1)as personas que se presenten a la investigación médica y/o sus sucesores deberán ser indemnizados por los daños ocasionados por la investigación médica. A falta de un sistema que asegure la indemnización a las personas perjudicadas, los Estados velarán porque se les otorguen las garantías suficientes para ser indemnizados. Las cláusulas que prevean excluir o limitar la indemnización de la víctima son nulas y no avenidas".

\section{LOS LÍMITES PERSONALES: LOS PRINCIPIOS}

\section{APLICABLES A LA PARTICIPACIÓN EN LA INVESTIGACIÓN BIOMÉDICA}

El principio de aceptabilidad. La unanimidad de los instrumentos internacionales insisten en la necesidad de que la participación en la investigación proyectada tenga que

\footnotetext{
45 Art. 24.

${ }^{46}$ Precisamente, el término “intervención” es el adoptado, con carácter general, por el Protocolo Adicional al Convenio sobre los Derechos Humanos y la Biomedicina de 25 de enero de 2005. Ver: art. 2. ${ }^{47}$ Art. 8.
} 
IUS ET SCIENTIA, (ISSN: 2444-8478) 2016, Vol.2, nº 1, pp.35-58

JOSÉ MANUEL SÁNCHEZ PATRÓN: LOS LÍMITES DE LA INVESTIGACIÓN BIOMÉDICA EN EL ORDENAMIENTO JURÍDICO INTERNACIONAL Y EUROPEO

FECHA DE ENVÍO DE ORIGINAL: 1.02.2016 FECHA DE ACEPTACIÓN: 1.03 .2016

DOI: http://dx.doi.org/10.12795/IETSCIENTIA.2016.i01.03

ser debidamente informada y convenientemente consentida de conformidad con el respeto a la autonomía de la persona a la que se le reconoce capacidad para tomar sus decisiones en relación con su participación en una investigación ${ }^{48}$. El Protocolo Adicional al Convenio sobre los Derechos Humanos y la Biomedicina de 25 de enero de 2005 realiza el mayor desarrollo de estos dos aspectos si lo comparamos con los diferentes textos tanto internacionales como europeos existentes al respecto ${ }^{49}$.

Para empezar, la información que se proporciona al posible participante en la investigación debe ser adecuada, comprensible y figurar por escrito ${ }^{50}$. Con carácter general, la información facilitada debe recoger el objetivo perseguido con la investigación, su planificación, los riesgos y los beneficios que eventualmente pueda acarrearle y la opinión del comité ético emitida sobre el mismo. Además, la información proporcionada debe reunir los derechos y garantías que la ley interna reconozca al participante para su protección; en particular, debe figurar en los textos legales el derecho a no prestar su consentimiento o bien a retirarlo en cualquier momento, sin que por ello pueda ser objeto de ninguna forma de discriminación, especialmente en lo que respecta a su derecho a recibir cuidados médicos. Adicionalmente, la información general anterior debe ser completada con una más específica, propia de cada investigación proyectada y en función de cual sea la naturaleza y los objetivos que se persigue con la realización de ésta última ${ }^{51}$.

Posteriormente, la persona otorgará su consentimiento a participar en la investigación ${ }^{52}$. Este consentimiento debe ser claro, libre, expreso, específico y consignado por escrito ${ }^{53}$. Aunque resulta difícil estar completamente exento, los participantes en una investigación proyectada deben estar al margen de cualquier

\footnotetext{
${ }^{48}$ Guía para los Miembros de los Comités de Ética de Investigación, op.cit., p. 10.

49 TORRES CAZORLA, M.I., GARCÍA RICO, E.M., El principio de libre autonomía de la persona: una primera aproximación desde la perspectiva del bioderecho internacional, en "Marco jurídico europeo relativo a la investigación biomédica en transferencia nuclear y reprogramación celular", op.cit., pp. 179-204 y FORNI, F., Consenso informato e sperimentazione clínica nella normativa dell'Unione Europea, en Diritto comunitairo e degli scambi internazionali, vol. 52, no 2, pp. 215-242.

${ }^{50}$ Art. 13.1 y 13.3 .

${ }^{51}$ Art. 13.2.

${ }^{52}$ El artículo 7 del Pacto Internacional de Derechos Civiles y Políticos de 16 de diciembre de 1966 establece que: "nadie será sometido sin su libre consentimiento a experimentos médicos o científicos".

${ }^{53}$ SALVATERRA, M., Informed consent to collect, store and use human biological materials for research purposes: an international framework, en "Trust and biobank: dealing with ethical, legal and social issues in a emerging field of biotechnology” (DABROCK, P.,), Springer, Berlin, pp. 127-137.
} 
IUS ET SCIENTIA, (ISSN: 2444-8478) 2016, Vol.2, nº 1, pp.35-58

JOSÉ MANUEL SÁNCHEZ PATRÓN: LOS LÍMITES DE LA INVESTIGACIÓN BIOMÉDICA EN EL ORDENAMIENTO JURÍDICO INTERNACIONAL Y EUROPEO

FECHA DE ENVÍO DE ORIGINAL: 1.02.2016 FECHA DE ACEPTACIÓN: 1.03 .2016

DOI: http://dx.doi.org/10.12795/IETSCIENTIA.2016.i01.03

obligación y/o influencia que les pueda llevar a aceptar - mediante incentivos, amenazas o cualquier otro medio de presión - un riesgo mayor del que sería aceptable por ellos. Esta obligación y/o influencia puede tener un origen diverso (económica, social, etc...) y dirigirse sobre los propios participantes o, incluso, sobre familiares cercanos ${ }^{54}$. Pese haber otorgado el consentimiento, el participante podrá retirarlo en cualquier estadio de la ejecución de la investigación, teniendo en cuenta que, tal y como veremos en relación con el principio de igualdad, no podrá ser discriminado por ello; en particular que su negativa sirva de pretexto para que se le deje de proporcionar cuidados médicos.

El principio que denominamos de aceptabilidad puede experimentar variaciones en su contenido y alcance dependiendo de las categorías de personas que participan en la investigación proyectada, así como de los tipos de situaciones en las que los participantes se van a encontrar. Los instrumentos internacionales prevén estas excepcionalidades que requieren de soluciones jurídicas especiales. En el presente trabajo, nos referiremos a ciertas categorías de participantes individuales - incapaces, embarazadas/lactantes - y colectivos; así como a diversos tipos de situaciones privación de libertad y urgencias clínicas - en las que pueden encontrarse ${ }^{55}$.

El principio de aceptabilidad ante determinada categorías de participantes: Los participantes individuales:

-Los individuos incapaces. El consentimiento informado es fundamental para participar en un proyecto de investigación. Sin embargo, la participación de individuos incapaces de consentir en este tipo de proyectos es igualmente importante para intentar dar respuesta a las enfermedades de este grupo. Por este motivo, es necesario encontrar

\footnotetext{
${ }^{54}$ Guía para los Miembros de los Comités de Ética de Investigación, op.cit., p. 11.

55 Aunque no se aplica a la investigación biomédica, el Protocolo Adicional al Convenio de Oviedo relativo a los test genéticos con fines médicos de 27 de noviembre de 2008 (art. 2 e Informe explicativo, p. 5, par. 32), contempla la posibilidad de realizar test genéticos a dos tipos de individuos: los desaparecidos y los fallecidos. Tanto en un caso como en el otro, la legislación permite, con ciertas condiciones, la realización de este tipo de pruebas en beneficio de "los miembros de una familia". Ver, arts. 14 y 15. Con este mismo objetivo y cumpliendo, también, condiciones específicas, los test genéticos pueden practicarse igualmente a personas incapaces de consentir. Art. 13. España no ha firmado ni ratificado este instrumento internacional por lo que no se encuentra en vigor para nuestro país.
} 
IUS ET SCIENTIA, (ISSN: 2444-8478) 2016, Vol.2, nº 1, pp.35-58

JOSÉ MANUEL SÁNCHEZ PATRÓN: LOS LÍMITES DE LA INVESTIGACIÓN BIOMÉDICA EN EL ORDENAMIENTO JURÍDICO INTERNACIONAL Y EUROPEO

FECHA DE ENVÍO DE ORIGINAL: 1.02.2016 FECHA DE ACEPTACIÓN: 1.03.2016

DOI: http://dx.doi.org/10.12795/IETSCIENTIA.2016.i01.03

la vía para facilitar su participación en un proyecto de investigación, supliendo la incapacidad de la que adolecen de otorgar al mismo su consentimiento informado ${ }^{56}$.

El Protocolo Adicional relativo a la investigación biomédica de 25 de enero de 2005 agrupa los requisitos que los textos internacionales recogen en relación con la participación de personas incapaces en proyectos de investigación científica ${ }^{57}$. Para empezar, como primer requisito, la participación de personas incapaces en la investigación proyectada debe ser necesaria. Esto quiere decir que si el proyecto de investigación puede efectuarse -estando garantizada una "efectividad comparable" con personas capaces, la participación de los que no lo sean debe descartarse. En segundo lugar, la persona incapaz debe ser informada de su participación en la investigación, especificándole sus “derechos y garantías" salvo que - debido a su incapacidad - no esté en condiciones de recibir esta información. En tercer lugar, la decisión de participar en la investigación proyectada debe adoptarla el representante, autoridad, persona o instancia que el derecho estatal establezca en cada caso concreto para suplir la incapacidad de la persona concernida. La prestación del consentimiento se hará por escrito y de forma específica. Para ello, quien le supla en la prestación del consentimiento debe estar debidamente informado del proyecto de investigación a realizar - según hemos visto al examinar el principio de aceptabilidad ${ }^{58}$-, a la vez que debe tomar en consideración "los deseos u objeciones" expresados por la persona incapaz acerca de su participación en el mismo. Lógicamente, este último extremo dependerá de las posibilidades que tenga la persona incapaz de comprender el alcance de su participación y aceptarla en consecuencia. No obstante, la normativa internacional exige que, cuando la persona incapaz sea mayor de edad, ésta, "en la medida de lo posible", se le debe "asociar al procedimiento de prestación del consentimiento" por parte de la persona autorizada para ello. Por el contrario, cuando la persona incapaz es un menor de edad, el parecer de éste último será tomado en consideración "como un factor, cada vez más determinante, en función de su edad así como de su grado de madurez". Todo ello sin olvidar que, como cuarto requisito, la persona incapaz no se oponga a su participación en el proyecto de investigación. Esta última condición,

\footnotetext{
${ }^{56}$ Guía para los Miembros de los Comités de Ética de Investigación, op.cit., p. 45.

${ }^{57}$ Estos principios y condiciones se encuentran sintetizados con respecto a la investigación genética en la Declaración Universal sobre el Genoma Humano y los Derechos Humanos de 11 de noviembre de 1997.

${ }^{58}$ Ver: art. 16 del Protocolo Adicional relativo a la investigación biomédica de 25 de enero de 2005.
} 
IUS ET SCIENTIA, (ISSN: 2444-8478) 2016, Vol.2, nº 1, pp.35-58

JOSÉ MANUEL SÁNCHEZ PATRÓN: LOS LÍMITES DE LA INVESTIGACIÓN BIOMÉDICA EN EL ORDENAMIENTO JURÍDICO INTERNACIONAL Y EUROPEO

FECHA DE ENVÍO DE ORIGINAL: 1.02.2016 FECHA DE ACEPTACIÓN: 1.03.2016

DOI: http://dx.doi.org/10.12795/IETSCIENTIA.2016.i01.03

examinada a la luz de la anterior, nos permite deducir que la decisión de participar en un proyecto de investigación le corresponde a la persona que el derecho interno habilite en los casos de personas incapaces. Esta persona autorizada podrá prestar el consentimiento preceptivo siempre y cuando la opinión de la persona incapaz, expresada en la medida de sus posibilidades, sea tomada en consideración. Ahora bien, si la persona incapaz se niega a participar en el proyecto de investigación, no se le podrá obligar a ello. Por último, y como quinto y último requisito, los resultados esperados con la realización del proyecto de investigación deben comportar un beneficio que pueda considerarse "real y directo" para la salud del incapaz ${ }^{59}$.

Los instrumentos internacionales han flexibilizado la exigibilidad del último requisito apuntado. La repercusión beneficiosa para la persona incapaz puede exceptuarse siempre y cuando se reúnan un par de requisitos adicionales que se suman a los cuatro primeros enunciados con anterioridad. En primer término,

"la investigación tenga por objeto contribuir, mediante una mejora significativa del conocimiento científico del estado de la persona, de su enfermedad o de su trastorno, a lograr en un determinado plazo resultados que permitan obtener un beneficio para la persona afectada o para otras personas de la misma categoría de edad o que padezcan la misma enfermedad o el mismo trastorno, o que presenten las mismas características" $"$.

Asimismo, y en segundo lugar,

"la investigación no presente para la persona concernida más que un riesgo mínimo o un inconveniente mínimo; cualquier consideración en cuanto a la importancia de los beneficios potenciales de la investigación no deber ser utilizada para justificar un nivel de riesgo o de los inconvenientes mayor" ${ }^{\prime \prime}$.

\footnotetext{
59 Art. 15.1.

${ }^{60}$ Art. 15.2 i).

${ }^{61}$ Art. 15.2 ii). El propio Protocolo Adicional relativo a la investigación biomédica de 25 de enero de 2005, clarifica que debe entenderse por "riesgo e inconveniente mínimo" en su artículo 17.
} 
IUS ET SCIENTIA, (ISSN: 2444-8478) 2016, Vol.2, nº 1, pp.35-58

JOSÉ MANUEL SÁNCHEZ PATRÓN: LOS LÍMITES DE LA INVESTIGACIÓN BIOMÉDICA EN EL ORDENAMIENTO JURÍDICO INTERNACIONAL Y EUROPEO

FECHA DE ENVÍO DE ORIGINAL: 1.02.2016 FECHA DE ACEPTACIÓN: 1.03.2016

DOI: http://dx.doi.org/10.12795/IETSCIENTIA.2016.i01.03

-Las embarazadas/lactantes. La investigación biomédica en mujeres embarazadas es necesaria para el conocimiento de las enfermedades relacionadas con este estado de salud que puedan afectar a la mujer, al embrión, al feto, al recién nacido o a todos ellos $^{62}$. El Protocolo Adicional relativo a la investigación biomédica de 25 de enero de 2005 dispone que la mujer embarazada puede participar en un proyecto de investigación si sus resultados previsibles son beneficiosos para "su salud, la del embrión, el feto o el recién nacido" ${ }^{\circ 3}$. Ahora bien ¿qué ocurre si la mujer embarazada pretende participar en una investigación proyectada cuyos resultados no tienen visos de serles beneficiosos a los destinatarios mencionados? En principio, en este caso, la mujer embarazada no podría participar. Sin embargo, el Protocolo Adicional examinado apunta tres condiciones que, si llegarán a reunirse, posibilitarían su participación en dicho proyecto de investigación aunque, a priori, no resultara beneficioso. La primera condición es que la investigación proyectada previera un resultado beneficioso para otras mujeres en relación con la procreación, para los embriones, los fetos o los recién nacidos. La segunda condición exige que la investigación de "eficacia comparable" no pueda llevarse a efecto en mujeres que no estén embarazadas. Y por último, como tercera condición, la ejecución del proyecto de investigación debe conllevar "un riesgo mínimo o un inconveniente mínimo" "64. No obstante, y en relación con ésta última condición, el Protocolo Adicional enfatiza que cuando participan en la investigación proyectada mujeres lactantes,

“(...) un cuidado especial debe ser adoptado para evitar eventuales efectos indeseables sobre la salud del recién nacido" ${ }^{\circ 5}$.

Con esta cautela, el tratado internacional analizado pone de manifiesto el interés especial concedido a la protección de la salud del "recién nacido" frente al otorgado con carácter general a la de la madre, el embrión o, incluso, a la del feto.

\footnotetext{
${ }^{62}$ Guía para los Miembros de los Comités de Ética de Investigación, op.cit., p. 51.

63 Este mismo tratado internacional dispone que cuando el proyecto de investigación prevé la participación de personas en "edad de procrear", “(...) una atención particular debe ser tomada con respecto a los eventuales efectos indeseables sobre una hipotético embarazo en curso o futuro y sobre la salud del embrión, el feto o el recién nacido". Art. 22.1.

${ }^{64}$ Art. 18.1. También, ver: art. 17.

${ }^{65}$ Art. 18.2.
} 
IUS ET SCIENTIA, (ISSN: 2444-8478) 2016, Vol.2, nº 1, pp.35-58

JOSÉ MANUEL SÁNCHEZ PATRÓN: LOS LÍMITES DE LA INVESTIGACIÓN BIOMÉDICA EN EL ORDENAMIENTO JURÍDICO INTERNACIONAL Y EUROPEO

FECHA DE ENVÍO DE ORIGINAL: 1.02.2016 FECHA DE ACEPTACIÓN: 1.03.2016

DOI: http://dx.doi.org/10.12795/IETSCIENTIA.2016.i01.03

El principio de aceptabilidad ante determinada categorías de participantes: Los participantes colectivos. Aunque el Protocolo Adicional al Convenio sobre los Derechos Humanos y la Biomedicina de 25 de enero de 2005 regula de una forma bastante completa este principio que llamamos de aceptabilidad, la Declaración Universal sobre Bioética y Derechos Humanos, de 19 de octubre de 2005, introduce un añadido que nos parece de interés mencionar, sobre todo, para los casos de investigación genética. Hasta ahora, lo dicho en relación con este principio está referido a la información facilitada $y$ al consentimiento requerido al participante en la investigación individualmente considerada. Pero, ¿qué ocurre cuando la investigación proyectada prevé la participación de un grupo o un colectivo de personas? Según la Declaración Universal referida, “(e)n los casos correspondientes a investigaciones llevadas a cabo en un grupo de personas o una comunidad, se podrá pedir además el acuerdo de los representantes legales del grupo o comunidad en cuestión" la disposición citada introduce una matización a destacar: "(e)l acuerdo colectivo de la comunidad o el consentimiento de un dirigente comunitario $\mathrm{u}$ otra autoridad no deberían sustituir en caso alguno el consentimiento informado del individuo"67.

El principio de aceptabilidad ante determinado tipo de situaciones:

-Los individuos privados de libertad. Los individuos pueden ser privados de libertad por motivos de seguridad, pero también por motivos de salud (estas personas pueden suponer un peligro para sus vidas o las de los demás). Esta privación de libertad los convierte en un grupo especialmente vulnerables como partícipes de un proyecto de investigación debido a su dependencia de otros para obtener alimentos, asistencia $\mathrm{u}$ otras necesidades, lo que puede dar lugar a que sean sometidos a abusos ${ }^{68}$. Sin embargo, esta vulnerabilidad no debería ser un obstáculo para que también puedan participar en investigaciones proyectadas, sobre todo, si éstas pueden redundar beneficiosamente en su salud. Por tal motivo, la Recomendación (1990)3 del Comité de Ministros del Consejo de Europa sobre la investigación médica sobre el ser humano de 6 de febrero de 1990 estable, aunque de forma lacónica, el siguiente principio:

\footnotetext{
${ }^{66}$ Art. 6.3

${ }^{67}$ Ibid.

${ }^{68}$ Guía para los Miembros de los Comités de Ética de Investigación, op.cit., p. 49.
} 
“(1)as personas privadas de libertad no pueden ser objeto de una investigación médica a no ser que obtengan un beneficio directo para su salud"69.

Este mismo principio es retomado por el artículo 20 del Protocolo Adicional relativo a la investigación biomédica de 25 de enero de 2005, si bien añade que la ley interna debe admitir la participación en la investigación proyectada, con lo que deja su aplicación en manos de los Estados Partes ${ }^{70}$. Esto quiere decir que, en la práctica, la participación de un individuo privado de libertad en un proyecto de investigación estará supeditada a éste sea beneficioso para su salud y que dicha participación esté contemplada en la legislación interna aplicable. Junto a ello, El Protocolo adicional establece una serie de garantías adicionales: la primera exige que no sea factible llevar a cabo la investigación proyectada con una "eficacia comparable" sin la participación de individuos privados de libertad. La segunda, que el proyecto de investigación tenga por objeto contribuir a la consecución de resultados capaces de proporcionar, en última instancia, un beneficio para las personas privadas de libertad. Y, la tercera, que el proyecto de investigación implique un riesgo o inconveniente mínimo ${ }^{71}$. Con las dos primeras garantías se quiere evitar que los individuos privados de libertad puedan ser explotados en beneficio de los que no lo estén, mientras que la tercera busca limitar la investigación para que no sobrepase los riesgos e inconvenientes mínimos ${ }^{72}$.

-Los individuos ante urgencias clínicas. El Convenio relativo a los Derechos Humano y la Biomedicina de 4 de abril de 1997 dispone que cualquier intervención médica, considerada favorable a la salud del individuo y de carácter indispensable, podrá realizarse sin necesidad de que se obtenga el "consentimiento adecuado" si se practica en "una situación de urgencia" "73. No obstante, en este supuesto, los deseos expresados por el paciente con anterioridad a dicha intervención médica deberán tomarse en consideración ${ }^{74}$. Ahora bien, este principio válido para las intervenciones médicas, ¿resulta extensible igualmente a las actuaciones ligadas a la investigación científica? El Protocolo Adicional relativo a la investigación biomédica de 25 de enero

\footnotetext{
${ }^{69}$ Principio 7.

${ }^{70}$ Ver: supra, nota 13

${ }^{71}$ Art. 20. También, ver: art. 17.

${ }^{72}$ Guía para los Miembros de los Comités de Ética de Investigación, op.cit., p. 51.

${ }^{73}$ Art. 8.

${ }^{74}$ Art. 9.
} 
IUS ET SCIENTIA, (ISSN: 2444-8478) 2016, Vol.2, nº 1, pp.35-58

JOSÉ MANUEL SÁNCHEZ PATRÓN: LOS LÍMITES DE LA INVESTIGACIÓN BIOMÉDICA EN EL ORDENAMIENTO JURÍDICO INTERNACIONAL Y EUROPEO

FECHA DE ENVÍO DE ORIGINAL: 1.02.2016 FECHA DE ACEPTACIÓN: 1.03 .2016

DOI: http://dx.doi.org/10.12795/IETSCIENTIA.2016.i01.03

de 2005 considera que nos encontramos ante un proyecto de investigación y una situación de urgencia clínica cuando la persona que participa en el mismo no ha podido dar su consentimiento y que en razón de dicha situación de urgencia no es posible obtener la autorización de quien le represente ${ }^{75}$. Dicho esto, ¿un individuo puede participar en una investigación proyectada en este supuesto de urgencia clínica? Realmente, el Protocolo Adicional deja la contestación a la pregunta formulada a lo que determine al respecto cada una de las legislaciones nacionales de los distintos Estados Partes. Por tanto, si el derecho interno lo permite, un proyecto de investigación podrá ejecutarse en una situación de urgencia clínica; en caso contrario, la investigación proyectada estará prohibida en estos casos excepcionales ${ }^{76}$.

No obstante, el Protocolo Adicional establece una serie de condiciones que la legislación nacional deberá observar caso de que el Estado Parte opte por permitir la realización de proyectos de investigación en supuestos de urgencia clínica. Las condiciones establecidas por este tratado internacional son las siguientes:

“i) una investigación de eficacia comparable no pueda ser efectuada sobre personas que no se encuentren en situación de urgencia; ii) el proyecto de investigación haya sido aprobado específicamente para situaciones de urgencia por la autoridad competente; iii) cualquier objeción pertinente expresada por la persona y puesta en conocimiento del investigador sea respetada; iv) si los resultados esperados de la investigación no comportan un beneficio directo para la salud de la persona concernida, la investigación tenga por objetivo contribuir, al menos, a una mejora significativa del conocimiento científico de su estado, de su enfermedad o de su trastorno, con la obtención, en su caso, de resultados que permitan un beneficio para la persona concernida o para otras personas de la misma categoría, o que padezcan la misma enfermedad o trastorno, o bien presenten el mismo estado de salud, siempre y cuando la investigación presente para la persona concernida un riesgo mínimo o un inconveniente mínimo" $" 77$.

\footnotetext{
75 Art. 19.1.

${ }^{76}$ Ver: supra, notas 13 y 70.

${ }^{77}$ Art. 19.2. También, ver: art. 17.
} 
IUS ET SCIENTIA, (ISSN: 2444-8478) 2016, Vol.2, nº 1, pp.35-58

JOSÉ MANUEL SÁNCHEZ PATRÓN: LOS LÍMITES DE LA INVESTIGACIÓN BIOMÉDICA EN EL ORDENAMIENTO JURÍDICO INTERNACIONAL Y EUROPEO

FECHA DE ENVÍO DE ORIGINAL: 1.02.2016 FECHA DE ACEPTACIÓN: 1.03 .2016

DOI: $\underline{\text { http://dx.doi.org/10.12795/IETSCIENTIA.2016.i01.03 }}$

En cualquier caso, el Protocolo Adicional añade a las condiciones anteriores que la realización del proyecto de investigación en situaciones de urgencia clínica no deba obstar para que el participante o su representante reciban la información correspondiente en el momento en el que sea posible y con independencia de su grado de materialización. A partir de este momento, se entiende que será necesario contar con el consentimiento de cualquiera de ellos para continuar con su ejecución ${ }^{78}$. Caso de no otorgarse dicho consentimiento o autorización, el participante o su representante deberían tener la posibilidad de solicitar que cualquier dato personal que ya hubiese sido recogido con anterioridad se retirase de la investigación ${ }^{79}$.

El principio de gratuidad. La Declaración de Helsinki de la Asociación Médica Mundial exige que para participar en una investigación, la persona debe hacerlo voluntariamente $^{80}$, sin esperar - tal y como señala la Recomendación (1990)3 del Comité de Ministros del Consejo de Europa sobre la investigación médica sobre el ser humano de 6 de febrero de 1990 - que se le retribuya económicamente por ello ${ }^{81}$. Según esta última Recomendación, “(1)as personas que se presten a la investigación médica no deben obtener por ello ningún beneficio económico" ${ }^{82}$. No obstante, una vez establecida la gratuidad de la participación en la investigación proyectada ${ }^{83}$, dicha Recomendación admite que: "los gastos realizados y las pérdidas ocasionadas pueden serles reembolsadas y, en su caso, una pequeña compensación puede serle concedida por los inconvenientes ocasionados por la investigación médica" ${ }^{\text {4 }}$. El reembolso de los gastos y las pérdidas generadas no puede constituir una "proporción sustancial de los ingresos o la única fuente de ingresos para los participantes"85. Asimismo, la compensación que se les otorgue por dichos inconvenientes tampoco puede ser tal que

\footnotetext{
78 Art. 19.3.

${ }^{79}$ Guía para los Miembros de los Comités de Ética de Investigación, op.cit., p. 48.

${ }^{80}$ B.20.

${ }^{81}$ También, ver: Declaración internacional sobre los Datos Genéticos Humanos de 16 de septiembre de 2003 , art. $8^{a}$ ).

82 Principio 13.

${ }^{83}$ Por lo que respecta a los investigadores, la Guía para los Miembros de los Comités de Ética de Investigación dispone que: "(l)os investigadores deberán dar detalles de cualquier pago, compensación o bienes materiales que les sean proporcionados a ellos o a su institución a cambio de la investigación". Op.cit., p. 43.

${ }^{84}$ Principio 13.

${ }^{85}$ Guía para los Miembros de los Comités de Ética de Investigación, op.cit., p. 43.
} 
IUS ET SCIENTIA, (ISSN: 2444-8478) 2016, Vol.2, nº 1, pp.35-58

JOSÉ MANUEL SÁNCHEZ PATRÓN: LOS LÍMITES DE LA INVESTIGACIÓN BIOMÉDICA EN EL ORDENAMIENTO JURÍDICO INTERNACIONAL Y EUROPEO

FECHA DE ENVÍO DE ORIGINAL: 1.02.2016 FECHA DE ACEPTACIÓN: 1.03 .2016

DOI: http://dx.doi.org/10.12795/IETSCIENTIA.2016.i01.03

les suponga una especie de "incentivo" que les empuje a "asumir un riesgo que (los) participante(s) no aceptarían en otras condiciones" diferentes ${ }^{86}$.

El principio de igualdad. Este principio considerado en su vertiente de prohibición de la discriminación resulta especialmente relevante en las investigaciones en las que se utilicen "información identificable”. La Declaración Internacional sobre los Datos Genéticos Humanos de 16 de septiembre de 2003 prevé que este tipo de datos no pueden utilizarse con fines que discriminen o que provoquen la estigmatización de "una persona, una familia, un grupo o comunidades", especialmente si se trata de estudios de genética de poblaciones o de genética del comportamiento ${ }^{87}$.

El Consejo Económico y Social de las Naciones Unidas se ha hecho eco de la problemática planteada en el ámbito concreto de la investigación y ha adoptado una Resolución titulada "Privacidad genética y no discriminación" de 21 de julio de 2004, en la que se "(i)nsta a los Estados a que siga apoyando la investigación en la esfera de la genética humana, con sujeción a normas científicas y éticas aceptadas y teniendo presentes los posibles beneficios para todas las personas, haciendo hincapié en que en esa investigación y en sus aplicaciones se deben respetar plenamente los derechos humanos, las libertades fundamentales y la dignidad humana, así como la prohibición de todas las formas de discriminación basada en rasgos genéticos" $" 88$.

El principio de confidencialidad. La Recomendación (1990)3 del Comité de Ministros del Consejo de Europa sobre la investigación médica sobre el ser humano de 6 de febrero de 1990, dispone que: “(t)oda información de carácter personal recogida con ocasión de una investigación médica sobre la persona que se preste debe ser considerada como confidencial" ${ }^{\prime 9}$. Este mismo principio ha sido reproducido en la Declaración Universal sobre el Genoma Humano y los Derechos Humanos, de 11 de noviembre de $1997^{90}$, el Protocolo Adicional al Convenio sobre los Derechos Humanos

\footnotetext{
${ }^{86}$ Ibid.

${ }^{87}$ Art. 7. También ver: Comité Director para la Bioética del Consejo de Europa. Informe del Grupo de Trabajo sobre la Genética Humana de 27 de octubre de 1997, especialmente, p. 25.

${ }^{88}$ Párr. $7^{\circ}$.

${ }^{89}$ Principio 9.

${ }^{90}$ Art. 7.
} 
IUS ET SCIENTIA, (ISSN: 2444-8478) 2016, Vol.2, nº 1, pp.35-58

JOSÉ MANUEL SÁNCHEZ PATRÓN: LOS LÍMITES DE LA INVESTIGACIÓN BIOMÉDICA EN EL ORDENAMIENTO JURÍDICO INTERNACIONAL Y EUROPEO

FECHA DE ENVÍO DE ORIGINAL: 1.02.2016 FECHA DE ACEPTACIÓN: 1.03 .2016

DOI: http://dx.doi.org/10.12795/IETSCIENTIA.2016.i01.03

y la Biomedicina, de 25 de enero de $2005^{91}$, y la Declaración Universal sobre Bioética y Derechos Humanos, de 19 de octubre de $2005^{92}$. La Declaración Internacional sobre los Datos Genéticos Humanos, de 16 de septiembre de 2003, ha clarificado y desarrollado este principio $^{93}$. Por lo que la investigación genética se refiere ${ }^{94}$, este instrumento internacional establece como regla general que los datos genéticos no "deberían" subrayamos la condicionalidad de la conjugación verbal - estar asociados a una persona concreta (información identificable) ${ }^{95}$. Y, aún, si así fuese, no “deberían” dejar de adoptarse las precauciones necesarias para garantizar la seguridad de los mismos en el desarrollo de la investigación ${ }^{96}$. Ahora bien, si los datos genéticos constituyen una información identificable, pese a que no es lo deseable, se podrá excepcionar la regla anterior “(...) cuando ello sea necesario para llevar a cabo la investigación, y a condición de que la privacidad de la persona y la confidencialidad de los datos o las muestras biológicas en cuestión queden protegidas con arreglo al derecho interno"97. Además, los participantes deben haber sido informados al respecto - grado de identificabilidad y quien tendrá acceso a los identificadores -, así como aceptar que la información identificable se utilice con los respectivos identificadores ${ }^{98}$.

En el ámbito de la Unión Europea, la Comisión Europea ha presentado una propuesta de Reglamento del Parlamento Europeo y del Consejo relativo a la protección de las personas físicas en lo que respecta al tratamiento de datos personales y a la libre circulación de estos datos ${ }^{99}$. Esta propuesta contiene una disposición específica sobre el

\footnotetext{
${ }^{91}$ Art. 25.

${ }^{92}$ Art. 9.

${ }^{93}$ Ver: nuestro trabajo: El régimen jurídico europeo aplicable a la confidencialidad de los datos relativos a la salud de las personas, en La salud: intimidad y libertades informativas (TOMAS Y VALIENTE LANUZA, C., dir.), Tirant lo Blanch, 2006, Valencia, 209-242.

94 NAVARRO CABALLERO, T.M., Investigación biomédica, tratamiento de muestras genéticas y biobancos, en "Bioderecho.es: Revista Internacional de Investigación en Bioderecho", n 1, 2014, pp. 115 .

95 GARCÍA SAN JOSÉ, D.I., El Derecho a la intimidad de la información genética en Derecho internacional y europeo: un escenario de luces y sombras, en "Nuevas tendencias en la interpretación de los derechos fundamentales"(GIMENO SENDRA, V., REGUEIRO GARCÍA, M.T., coord.), Universitas, Madrid, 2015, pp. 321-338.

96 Art. $14 \mathrm{c}$ )

${ }^{97}$ Art. 14 d)

${ }^{98}$ Guía para los Miembros de los Comités de Ética de Investigación, op.cit., p. 40.

${ }^{99}$ Esta propuesta fue presentada por la Comisión Europea el 25 de enero de 2012. Documento $\operatorname{COM}(2012) 11$ final de 25 de enero de 2012 y con ella se pretende reemplazar la Directiva 95/46/CE del Parlamento Europeo y del Consejo, de 24 de octubre de 1995, relativa a la protección de las personas físicas en lo que respecta al tratamiento de datos personales y a la libre circulación de estos datos, que se encuentra actualmente en vigor. D.O.C.E n ${ }^{\circ}$ L281/31 de 23 de noviembre de 1995.
} 
IUS ET SCIENTIA, (ISSN: 2444-8478) 2016, Vol.2, nº 1, pp.35-58

JOSÉ MANUEL SÁNCHEZ PATRÓN: LOS LÍMITES DE LA INVESTIGACIÓN BIOMÉDICA EN EL ORDENAMIENTO JURÍDICO INTERNACIONAL Y EUROPEO

FECHA DE ENVÍO DE ORIGINAL: 1.02.2016 FECHA DE ACEPTACIÓN: 1.03 .2016

DOI: $\underline{\text { http://dx.doi.org/10.12795/IETSCIENTIA.2016.i01.03 }}$

tratamiento de datos personales relativos a la salud y que resultan necesarios para la investigación científica ${ }^{100}$. A tal efecto, el futuro Reglamento comunitario establece una serie de "condiciones y garantías" con respecto a este tipo de información; en particular, sobre su tratamiento y difusión ${ }^{101}$.

\section{CONCLUSIONES}

El contenido de los distintos apartados de este trabajo nos permite concluir que la investigación biomédica está sometida a una serie de límites que tienen como objetivo salvaguardar los derechos fundamentales del individuo. Esta finalidad explica que los beneficios generales que puedan extraerse de las investigaciones proyectadas para la sociedad estarán supeditados a los intereses particulares del individuo, de tal manera que, aunque resulte paradójico, aquellos deberán ser sacrificados ante la primacía de estos últimos. Esta prevalencia de los derechos humanos frente a los hipotéticos beneficios para la humanidad que puedan derivarse de las investigaciones científicas justifica las exigencias y cautelas a las que se somete está actividad según figuran en los diferentes instrumentos internacionales que hemos examinado. Todas ellas las hemos intentado sistematizar en forma principios agrupados en torno a la ejecución de un proyecto de investigación y la participación de los individuos en el mismo. Aunque estos principios deban adaptarse o, llegado el caso, incluso, desarrollarse en función de los nuevos retos que planteen las investigaciones futuras en el ámbito de la biomedicina,

\footnotetext{
${ }^{100}$ Art. 81.2 y 83.

${ }^{101}$ Según la propuesta del art. 83: "1.Dentro de los límites del presente Reglamento, podrán tratarse los datos personales para fines de investigación histórica, estadística o científica sólo si: a) dichos fines no pueden lograrse de otra forma mediante un tratamiento de datos que no permita o que ya no permita la identificación del interesado; b) los datos que permitan la atribución de información a un interesado identificado o identificable se conservan por separado del resto de la información, en la medida en que dichos fines puedan lograrse de este modo. 2. Los organismos que llevan a cabo investigaciones históricas, estadísticas o científicas podrán publicar o hacer públicos por otra vía datos personales sólo si:

a) el interesado ha dado su consentimiento en las condiciones establecidas en el artículo 7; b) la publicación de los datos personales es necesaria para presentar los resultados de una investigación o para facilitar una investigación, siempre que los intereses o los derechos o libertades fundamentales del interesado no prevalezcan sobre tales objetivos; o c) el interesado ha hecho públicos los datos. 3. La Comisión estará facultada para adoptar actos delegados, de conformidad con el artículo 86, a fin de especificar los criterios y requisitos del tratamiento de los datos personales a los efectos mencionados en los apartados 1 y 2, así como las limitaciones necesarias a los derechos de información y de acceso por parte del interesado, y de detallar las condiciones y garantías de los derechos del interesado en tales circunstancias".
} 
IUS ET SCIENTIA, (ISSN: 2444-8478) 2016, Vol.2, $\mathrm{n}^{\circ} 1$, pp.35-58

JOSÉ MANUEL SÁNCHEZ PATRÓN: LOS LÍMITES DE LA INVESTIGACIÓN BIOMÉDICA EN EL ORDENAMIENTO JURÍDICO INTERNACIONAL Y EUROPEO

FECHA DE ENVÍO DE ORIGINAL: 1.02.2016 FECHA DE ACEPTACIÓN: 1.03 .2016

DOI: $\underline{\text { http://dx.doi.org/10.12795/IETSCIENTIA.2016.i01.03 }}$

qué duda cabe que tales principios constituyen un faro para los investigadores que arroja luz acerca de cuáles son las posibilidades y los confines de sus investigaciones. Precisamente, con el presente trabajo hemos pretendido sintetizarlos y clarificarlos. 